\title{
Bayesian Analysis of Two Parameter Complementary Exponential Power Distribution
}

\author{
A.K.Chaudhary (Ph D) \\ Associate Professor, Nepal Commerce Campus
}

\begin{abstract}
:
In this paper, the Markov chain Monte Carlo (MCMC) method is used to estimate the parameters of CEP distribution based on a complete sample. A procedure is developed to obtain Bayes estimates of the parameters of the CEP distribution using Markov Chain Monte Carlo (MCMC) simulation method in OpenBUGS, established software for Bayesian analysis using Markov Chain Monte Carlo (MCMC) methods. The MCMC methods have been shown to be easier to implement computationally, the estimates always exist and are statistically consistent, and their probability intervals are convenient to construct. The $R$ functions are developed to study the statistical properties, model validation and comparison tools of the distribution and the output analysis of MCMC samples generated from OpenBUGS. A real data set is considered for illustration under uniform and gamma sets of priors.
\end{abstract}

Keywords: Bayesian estimation, Complementary exponential power distribution, Maximum likelihood estimation, Markov chain Monte Carlo, Model validation, OpenBUGS.

\section{Introduction}

The exponential power (EP) distribution was firstly introduced as a lifetime model by [Smith and Bain (1975)]. This distribution has been discussed by many authors [Leemis (1986)], [Rajarshi and Rajarshi(1988)] and [Chen (1999)].

In recent years, new classes of models have been proposed based on modifications of the EP model. [Chen (2000)] proposed a new model with two shape parameters. His model is appealing since though having only two parameters it can accommodate increasing and bathtub shaped hazard functions. Also, it holds some nice properties on the classical inferential front. The confidence intervals for the shape parameters and their joint confidence regions have closed form. However, it lacks a scale parameter that makes it less flexible for analyzing a variety of datasets. To overcome such a limitation, [Xie et al.(2002)] proposed a model, known as the Weibull extension model, which can be considered as an extension of Chen's model, with an additional scale 
parameter. As a result, the model becomes more flexible and persuasive from the point of view of practitioners. The Xie model version however accommodates only increasing and bathtub shaped hazard functions as its antecessors.

Although the EP distribution and its modifications are commonly used for analyzing lifetime data, they do not provide a reasonable parametric fit for some practical applications where the underlying hazard functions may be decreasing or unimodal shaped.

Recently, [Barriga et al. (2011)] introduced the Complementary Exponential Power(CEP) distribution, which is mainly related to the distribution proposed by [Smith and Bain (1975)] with an additional shape parameter.

In the survival literature there are several flexible distributions which can accommodate increasing, decreasing, unimodal and bathtub shaped hazard functions [Mudholkar and Srivastava(1993)], [Mudholkar et al.,(1996)], [Pham and Lai (2007)] and [Carrasco et al.(2008)]. Some of them are four-parameter distributions, while the CEP is a three-parameter one. This is an advantage from a practical point of view, since it is important to consider parsimonious models with as few parameters as possible. Particularly, with small and moderate sized samples, a usual situation in survival analysis, where the parameters may not be accurately estimated [Xie et al. (2002)].

As described by [Marshall and Olkin (2007)], an exponentiated distribution can be easily constructed. It is based on the observation that by raising any baseline cumulative distribution function (cdf) $\mathrm{F}_{\text {baseline }}(\mathrm{x})$ to an arbitrary power $\theta>0$, a new cdf $\mathrm{F}(\mathrm{x})=\left(\mathrm{F}_{\text {baseline }}(\mathrm{x})\right)^{\theta} ; \theta>0$ is obtained, but now with the additional parameter $\theta$, which can be referred as a resilience parameter and $\mathrm{F}(\mathrm{x})$ is a resilience parameter family. Although it is not our case, the term resilience easily emerges if we let $\theta$ be an integer. In this case $\mathrm{F}(\mathrm{x})$ can be seem as the cdf of a parallel system with $\theta$ independent components, which is less likely to fail as the number of components increases, leading to a resilient structure. Following this idea, several authors have considered extensions from usual survival distributions. For instance, [Mudholkar et al. (1995)] considered the exponentiated Weibull distribution as a generalization of the Weibull distribution, [Gupta and Kundu (1999)] introduced the exponentiated exponential distribution as a generalization of the usual exponential distribution and [Nadarajah and Kotz (2006)] proposed exponentiated type distributions extending the Frchet, gamma, Gumbel and Weibull distributions.

The cdf of the exponential power (EP) distribution proposed by [Smith and Bain (1975)] is given by

$$
F_{E P}(x ; \alpha, \lambda)=1-\exp \left\{1-\exp (\lambda x)^{\alpha}\right\} ; \quad \alpha, \lambda>0 ; x>0
$$

The cdf of the CEP distribution is defined by raising $\mathrm{F}_{\mathrm{EP}}(\mathrm{x})$ to the power of $\theta$ namely $\mathrm{F}(\mathrm{x})=\left(\mathrm{F}_{\mathrm{EP}}(\mathrm{x})\right)^{\theta}$. The distribution function of Complementary Exponential Power(CEP) distribution with three parameters is given by, [Barriga et al. (2011)]

$$
F(x ; \alpha, \lambda, \theta)=\left[1-\exp \left\{1-\exp (\lambda x)^{\alpha}\right\}\right]^{\theta} ; \quad \alpha, \lambda, \theta>0 ; x \geq 0
$$

where $\alpha>0$ and $\theta>0$ are shape parameters and $\lambda>0$ is a scale parameter.

$$
\sim 2 \sim
$$


When $\theta=1$ the model (1.1) reduces to the EP distribution. The CEP distribution will be denoted by $\operatorname{CEP}(\alpha, \lambda, \theta)$. If $\lambda=1$, we have two-parameter CEP distribution and we shall denote it as $\operatorname{CEP} 2(\alpha, \theta)$

The rest of the article is organized as follows. The model and its features are introduced in Section 2. In Section 3, we have discussed the Bayesian model formulation including the priors, posterior, Gibbs sampler and its implementation in OpenBUGS. The real data set and its exploratory data analysis, maximum likelihood estimation(MLE) and model validation are described in Section 4. The full Bayesian analysis under independent gamma of priors for the data set using Markov chain Monte Carlo (MCMC) simulation method in OpenBUGS, an established software, is presented in Section 5. The Bayes estimates of the parameters and their probability intervals based on posterior samples are presented. The posterior analysis is performed and we have also estimated the reliability function. Conclusions are given in Section 6.

\section{Complementary Two Parameter Exponential Power (CEP) dis- tribution}

\subsection{Model Analysis}

The cumulative distribution function of complementary exponential power(CEP) distribution with two parameters is given by

$$
F(x ; \alpha, \theta)=\left[1-\exp \left\{1-\exp \left(x^{\alpha}\right)\right\}\right]^{\theta} ; \alpha, \theta>0
$$

where $\alpha>0$ and $\theta>0$ are the shape parameters. The corresponding probability density function is given by

$$
\begin{gathered}
f(x ; \alpha, \theta)=\alpha \theta x^{\alpha-1} \exp \left(x^{\alpha}\right) \exp \left(1-\exp \left(x^{\alpha}\right)\right) \\
{\left[1-\exp \left(1-\exp \left(x^{\alpha}\right)\right)\right]^{\theta-1}}
\end{gathered}
$$
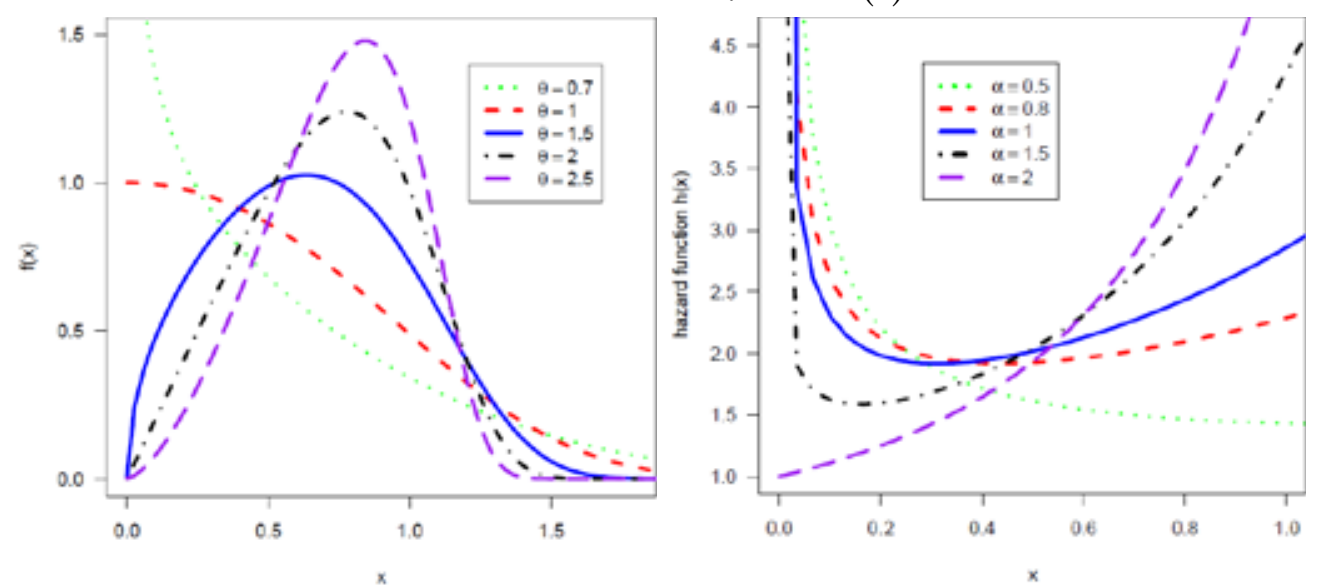

Figure 1 The probability density function (left); The hazard function (right) of $\operatorname{CEP} 2(\alpha, \theta)$ distribution for $\theta=1$ and different values of $\alpha$. 
The reliability/survival function is

$$
R(x ; \alpha, \theta)=1-\left[1-\exp \left\{1-\exp \left(x^{\alpha}\right)\right\}\right]^{\theta} ; \alpha, \theta>0
$$

The hazard rate function is

$$
h(x ; \alpha, \theta)=\frac{\alpha \theta x^{\alpha-1} \exp \left(x^{\alpha}\right) \exp \left[1-\exp \left(x^{\alpha}\right)\right] \cdot\left[1-\exp \left\{1-\exp \left(x^{\alpha}\right)\right\}\right]^{\theta-1}}{1-\left[1-\exp \left\{1-\exp \left(x^{\alpha}\right)\right\}\right]^{\theta}}
$$

The quantile function is given by

$$
x_{p}=\left[\ln \left\{1-\ln \left(1-p^{1 / \theta}\right)\right\}\right]^{1 / \alpha} ; 0<p<1 \text {. }
$$

The random deviate can be generated from

$$
C E P(\alpha, \theta) \text { by } x=\left[\ln \left\{1-\ln \left(1-u^{1 / \theta}\right)\right\}\right]^{1 / \alpha}
$$

where $\mathrm{u}$ has the $U(0,1)$ distribution

Some of the typical $C E P 2(\alpha, \theta)$ density functions for different values of $\alpha$ and for $\theta=1$ are depicted in Figure 1 (top). It is evident from the Figure 1 that the density function of the CEP distribution can take different shapes. Figure 1(bottom) exhibits the different hazard rate functions of $\operatorname{CEP} 2(\alpha, \theta)$ distribution.

The CEP distribution accommodates unimodal, bathtub and a broad variety of monotone hazard functions depending on the parameter values over the regions of the space of the shape parameters $\alpha>0$ and $\theta>0$, separated by the boundary $\theta=1, \alpha=1$ and the curve $\alpha \theta=1$. Continue to enumerate some properties of the hazard function.

i. $\quad \mathrm{h}(0)=0$, if $\alpha>1$, and $\mathrm{h}(0)=1$, if $\alpha=1$ and $\theta=1$,

ii. If $\alpha>1$ and $\theta \leq 1$, we have $\mathrm{h}(\mathrm{x})$ is increasing,

iii. If $\theta>1$ and $\alpha \theta \leq 1$, we have $\mathrm{h}(\mathrm{x})$ is decreasing,

iv. If $\alpha<1$ and $\alpha \theta>1$, we have $\mathrm{h}(\mathrm{x})$ is upside-down bathtub (unimodal),

v. If $\alpha<1$ and $\theta \leq 1$ (or $\alpha>1$ and $\alpha \theta<1$ ), we have $\mathrm{h}(\mathrm{x})$ is bathtub.

Since the failure rate function in (4) is very complex, some of the properties II-V were obtained numerically. The distinct types of hazard shapes are illustrated in Figure 1. for some different parameter combinations of the CEP distribution.

\section{Bayesian model formulation}

In this section, we provide the Bayes estimates of the shape parameters assuming independent gamma priors for both the parameters $\alpha$ and $\theta$. For the $C E P 2(\alpha, \theta)$, the Bayesian model is constructed by specifying a prior distribution for $\alpha$ and , and then multiplying with the likelihood function to obtain the posterior distribution function. Given a set of data $\underline{x}=\left(x_{1}, \ldots, x_{n}\right)$, the likelihood function is 


$$
\begin{aligned}
L(\alpha, \theta \mid \underline{x})=\alpha^{n} \theta^{n}\left(\prod_{i=1}^{n} x_{i}^{\alpha-1}\right) \exp \left(\sum_{i=1}^{n} x_{i}^{\alpha}+\sum_{i=1}^{n}\left(1-\exp \left(x_{i}^{\alpha}\right)\right)\right. \\
\left(\prod_{i=1}^{n}\left[1-\exp \left\{1-\exp \left(x_{i}^{\alpha}\right)\right\}\right]^{\theta-1}\right)
\end{aligned}
$$

Prior distributions

Denote the prior distribution of $\alpha$ and $\theta$ as $p(\alpha, \theta)$. The joint posterior is

$$
p(\alpha, \theta \mid \underline{x}) \propto L(\alpha, \theta \mid \underline{x}) p(\alpha, \theta) .
$$

We assume the independent gamma priors for $\alpha \sim G(a, b)$ and $\theta \sim G(c, d)$ as

$$
p(\alpha)=\frac{b^{a}}{\Gamma(a)} \alpha^{a-1} \mathrm{e}^{-b \alpha} ; \alpha>0,(a, b)>0
$$

and

$$
p(\theta)=\frac{d^{c}}{\Gamma(c)} \theta^{c-1} \mathrm{e}^{-d \theta} ; \theta>0,(c, d)>0 .
$$

\section{Posterior distribution}

Combining the likelihood function with the prior via Bayes' theorem yields the posterior up to proportionality as

$$
\begin{gathered}
\mathrm{p}(\alpha, \theta \mid \underline{\mathrm{x}}) \propto \exp \left(-\mathrm{b} \alpha-\mathrm{d} \theta+\sum_{\mathrm{i}=1}^{\mathrm{n}} \mathrm{x}_{\mathrm{i}}^{\alpha}+\sum_{\mathrm{i}=1}^{\mathrm{n}}\left(1-\exp \left(\mathrm{x}_{\mathrm{i}}^{\alpha}\right)\right)\right. \\
\alpha^{\mathrm{n}+\mathrm{a}-1} \theta^{\mathrm{n}+\mathrm{c}-1}\left(\prod_{\mathrm{i}=1}^{\mathrm{n}} \mathrm{x}_{\mathrm{i}}^{\alpha-1}\right) \mathrm{T}_{1}
\end{gathered}
$$

where $T_{1}=\prod_{i=1}^{n}\left[1-\exp \left\{1-\exp \left(x_{i}^{\alpha}\right\}\right]^{\theta-1}\right.$.

The posterior is obviously complicated and no close form inferences appear possible. We, therefore, propose to consider MCMC methods to simulate samples from the posterior so that sample-based inferences can be easily drawn.

The Gibbs sampler is as an important Markov Chain Monte Carlo technique, which provides a way for extracting samples from the posteriors.. This sampling scheme was first introduced by Geman and Geman(1984), but the applicability to statistical modelling for Bayesian computation was demonstrated by Gelfand and Smith (1990).

It generates a sample from an arbitrarily complex multidimensional distribution by sampling from each of the univariate full conditional distributions in turn. That is, every time a variate value is generated from a full conditional, it is influenced by the most recent values of all other conditioning variables and, after each cycle of iteration, it is updated by sampling a new value from its full conditional. The entire generating scheme is repeated unless the generating chain achieves a systematic pattern of convergence. It can be shown that after a large number of iterations the generated variates can be regarded as the random samples from the corresponding posteriors. Gelman et al. (2004), Albert (2009), Hamada et al.(2008), Ntzoufras (2009) and Hoff 
(2009) provide the details of the procedure and the related convergence diagnostic issues.

Therefore to obtain the full conditional distribution of $\alpha$ (or $\theta$ ), we need only choose the terms in the posterior, which involve parameter $\alpha$ (or $\theta$ ). The full posterior conditional distributions for and $\theta$ are

$$
\begin{aligned}
p(\alpha \mid \theta, \underline{x}) \propto \alpha^{n+a_{2}-1}\left(\prod_{i=1}^{n} x_{i}^{\alpha-1}\right) \\
\exp \left(-b_{1} \alpha+\sum_{i=1}^{n} x_{i}^{\alpha}+\sum_{i=1}^{n}\left(1-\exp \left(x_{i}^{\alpha}\right)\right) T_{1}\right.
\end{aligned}
$$

and

$$
p(\theta \mid \alpha, \underline{x}) \propto \theta^{n+a_{2}-1} \exp \left(-b_{2} \theta\right) T_{1} .
$$

As the complementary exponential power(CEP) distribution is not available in OpenBUGS., it requires incorporation of a module in ReliaBUGS, Kumar et al.(2010) and Lunn(2010), subsystem of OpenBUGS for CEP.

A module dcom.expo_T(alpha, theta) is written in Component Pascal for CEP, the corresponding computer program can be obtained from authors, to perform full Bayesian analysis in OpenBUGS using the method described in Thomas et al(2006), Thomas(2010), Kumar et al.(2010) and Lunn et al. (2013). It is important to note that this module can be used for any set of suitable priors of the model parameters. Almost all aspects of the model in Bayesian framework can be studied using the developed module dcom.expo_T(alpha, theta), Kumar(2010).

\section{Gibbs Sampler : Implementation}

1. Select an initial value $\underline{\delta}^{(0)}=\left(\alpha^{(0)}, \theta^{(0)}\right)$ to start the chain.

2. Suppose at the ith-step, $\underline{\delta}=(\alpha, \theta)$ takes the value $\underline{\delta}^{(i)}=\left(\alpha^{(i)}, \theta^{(i)}\right)$ then from full conditionals, we generate

$$
\begin{aligned}
& \alpha^{(i+1)} \text { from } p\left(\alpha \mid \theta^{(i)}, \underline{x}\right) \text { and } \\
& \theta^{(i+1)} \text { from } p\left(\theta \mid \alpha^{(i+1)}, \underline{x}\right) .
\end{aligned}
$$

3. This completes a transition from $\underline{\delta}^{(i)}$ to $\underline{\delta}^{(i+1)}$.

4. Repeat Step 2, N times.

\section{MCMC output : Posterior sample}

Monitor the convergence using convergence diagnostics(trace and ergodic mean plots). Suppose that convergence have been reached after 'B' iterations (the burn-in period). Discard the observations $\left(\underline{\delta}^{(1)}, \underline{\delta}^{(2)}, \ldots, \underline{\delta}^{(B)}\right)$ and retain the observations $\left(\underline{\delta}^{(B+1+(j-1) L)} ; B+1+(M-1) L \leq N ; j=1,2, \ldots, M ; L \geq 1\right)$ which are viewed as being an independent sample from the stationary distribution of the Markov chain that is typically the posterior distribution, where ' $\mathrm{L}$ ' is the lag (or thin interval).

Consider $\left(\underline{\delta}^{(1)}, \ldots, \underline{\delta}^{(j)}, \ldots, \underline{\delta}^{(M)}\right)$ as the MCMC output (posterior sample) for the 
posterior analysis

$$
\underline{\delta}^{(j)}=\left(\alpha^{(j)}, \theta^{(j)}\right) ; j=1,2, \ldots, M
$$

Thus MCMC output is referred as the sample after removing the initial iterations (produced during the burn-in period) and considering the appropriate lag.

The Bayes estimates of $\underline{\delta}=(\alpha, \theta)$ using the ergodic theorem are given by

$$
\hat{\alpha}=\frac{1}{M} \sum_{j=1}^{M} \alpha^{(j)} \quad \text { and } \hat{\theta}=\frac{1}{M} \sum_{j=1}^{M} \theta^{(j)} \text {. }
$$

An important advantage of sample-based approaches includes the routine developments for any linear and/or non-linear functions of the original parameters. It is to be noted that once the samples from the posterior is obtained, samples from the posterior of any linear and/or non-linear functions can be easily created merely by substitution. Some of such functions where reliability practitioners are often interested include reliability, hazard rate, mean time to failure, percentiles, etc.

\section{Data, Maximum Likelihood Estimation and Model validation}

The following real data set is considered for illustration of the proposed methodology. The data set is originally considered by Badar and Priest. The data given represent the strength measured in GPA for single carbon fibers of $10 \mathrm{mmin}$ gauge lengths with sample size 63 and they are as follows:

$1.901,2.132,2.203,2.228,2.257,2.350,2.361,2.396,2.397,2.445,2.454,2.474$, $2.518,2.522,2.525,2.532,2.575,2.614,2.616,2.618,2.624,2.659,2.675,2.738$, $2.740,2.856,2.917,2.928,2.937,2.937,2.977,2.996,3.030,3.125,3.139,3.145$, $3.220,3.223,3.235,3.243,3.264,3.272$, 3.294, 3.332, 3.346, 3.377, 3.408, 3.435, 3.493, 3.501, 3.537, 3.554, 3.562, 3.628, 3.852, 3.871, 3.886, 3.971, 4.024, 4.027, $4.225,4.395,5.020$

\subsection{Exploratory data analysis (EDA)}

EDA is an approach to statistical analysis, heavily graphical in nature that attempts to maximize insight into data, Tukey(1977). It allows data to speak for themselves, without making assumptions and conducting formal analyses. The descriptive statistical methods quantitatively describe the main features of data.

The main data features are (i) measures of central tendency(e.g. mean and median); (ii) measures of variability (e.g., standard deviation) and (iii) measures of relative standing (e.g., quantiles). The descriptive statistics for the above data set are presented in Table 1. We have plotted the boxplot in Figure 2, which shows that data set contains one "outlier". We have also plotted the histogram of real data set which shows positive skewed. 

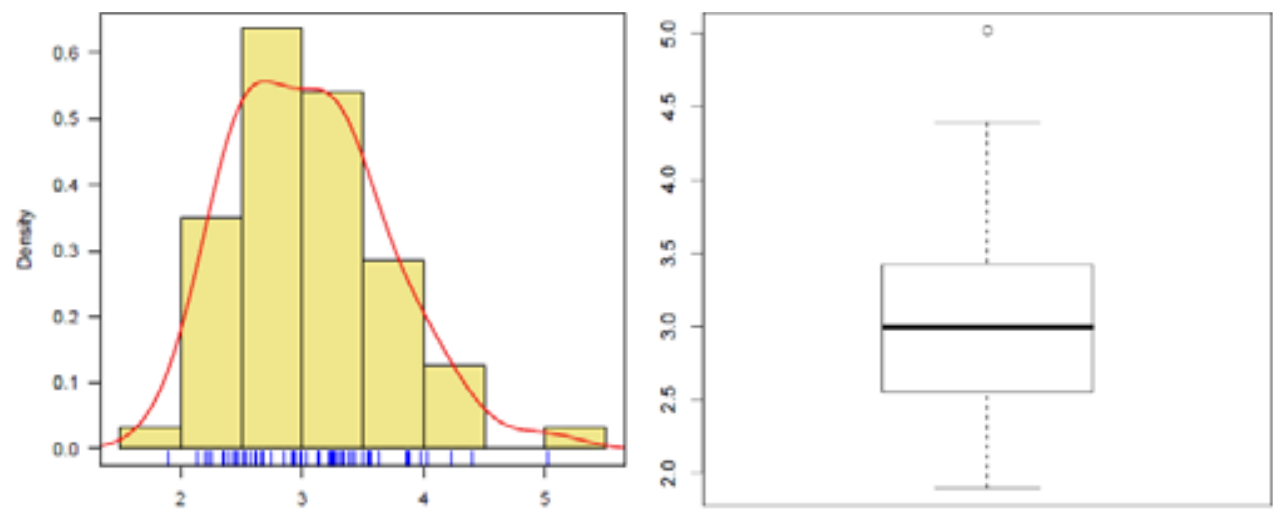

Figure 2. Histogram (left) and boxplot (right)

Table -1: Summary statistics

\begin{tabular}{|l|c|}
\hline Minimum & 0.200 \\
\hline First Quartile (Q1) & 0.800 \\
\hline Median & 1.750 \\
\hline Mean & 3.607 \\
\hline Third Quartile (Q3) & 4.375 \\
\hline Maximum & 24.500 \\
\hline Kurtosis & 8.295 \\
\hline Skewness & 2.795 \\
\hline
\end{tabular}

The estimation of the parameter of proposed model is obtained by the method of maximum likelihood(ML) estimation.

\subsection{Maximum likelihood estimation (MLE) and asymptotic confidence intervals}

For completeness purposes, in this section, we briefly discuss the maximum likelihood estimators (MLE's) of the two-parameter CEP distribution and discuss their asymptotic properties to obtain approximate confidence intervals based on MLE's.

Let $\underline{x}=\left(x_{1}, \ldots, x_{n}\right)$ be a random sample of size $\mathrm{n}$ from $C E P(\alpha, \theta)$, then the loglikelihood function $\ell(\alpha, \theta)$ can be written as;

$$
\begin{aligned}
\ell(\alpha, \theta \mid \underline{x})= & n \ln \alpha+n \ln \theta+(\alpha-1) \sum_{i=1}^{n} \ln \left(x_{i}\right)+\sum_{i=1}^{n} x_{i}^{\alpha}+n-\exp \left(\sum_{i=1}^{n} x_{i}^{\alpha}\right) \\
& +(\theta-1) \sum_{i=1}^{n} \ln \left[1-\exp \left(1-\exp \left(x_{i}^{\alpha}\right)\right]\right.
\end{aligned}
$$

Therefore, to obtain the MLE's of $\alpha$ and $\theta$, we can maximize (9) directly with respect to $\alpha$ and $\theta$ or we can solve the following two non-linear equations using iterative method e.g. Newton-Raphson method 


$$
\begin{aligned}
& \frac{\partial \ell}{\partial \alpha}=\frac{n}{\alpha}+ \sum_{i=1}^{n} \ln \left(x_{i}\right)+\sum_{i=1}^{n} x_{i}^{\alpha} \ln \left(x_{i}\right)-\sum_{i=1}^{n} \exp \left(x_{i}^{\alpha}\right) x_{i}^{\alpha} \ln \left(x_{i}\right) \\
&+(\theta-1) \sum_{i=1}^{n} \frac{x_{i}^{\alpha} \exp \left(x_{i}^{\alpha}\right) \exp \left(1-\exp \left(x_{i}^{\alpha}\right) \ln \left(x_{i}\right)\right.}{1-\exp \left\{1-\exp \left(x_{i}^{\alpha}\right)\right\}} \\
& \frac{\partial \ell}{\partial \theta}=\frac{n}{\theta}+\sum_{i=1}^{n} \ln \left[1-\exp \left(1-\exp \left(x_{i}^{\alpha}\right)\right]\right.
\end{aligned}
$$

Let us denote the parameter vector by $\underline{\delta}=(\alpha, \theta)$ and the corresponding MLE of $\underline{\delta}$ as $\underline{\hat{\delta}}=(\hat{\alpha}, \hat{\theta})$ then the asymptotic normality results in

$$
(\underline{\hat{\delta}}-\underline{\delta}) \rightarrow N_{2}\left(0,(I(\underline{\delta}))^{-1}\right)
$$

where $I(\underline{\delta})$ is the Fisher's information matrix given by

$$
I(\underline{\delta})=-\left[\begin{array}{ll}
E\left(\frac{\partial^{2} \ell}{\partial \alpha^{2}}\right) & E\left(\frac{\partial^{2} \ell}{\partial \alpha \partial \theta}\right) \\
E\left(\frac{\partial^{2} \ell}{\partial \theta \partial \alpha}\right) & E\left(\frac{\partial^{2} \ell}{\partial \theta^{2}}\right)
\end{array}\right] .
$$

In practice, it is useless that the MLE has asymptotic variance $(I(\underline{\delta}))^{-1}$ because we do not know $\underline{\delta}$. Hence, we approximate the asymptotic variance by "plugging in" the estimated value of the parameters. The common procedure is to use observed Fisher information matrix $O(\underline{\hat{\delta}})$ (as an estimate of the information matrix $I(\underline{\delta})$ ) given by

$$
O(\underline{\hat{\delta}})=-\left(\begin{array}{cc}
\frac{\partial^{2} \ell}{\partial \alpha^{2}} & \frac{\partial^{2} \ell}{\partial \alpha \partial \theta} \\
\frac{\partial^{2} \ell}{\partial \theta \partial \alpha} & \frac{\partial^{2} \ell}{\partial \theta^{2}}
\end{array}\right)_{(\hat{\alpha}, \hat{\theta})}=-\left.H(\delta)\right|_{\underline{\delta}=\underline{\hat{\delta}}}
$$

where $\mathrm{H}$ is the Hessian matrix, $\underline{\delta}=(\alpha, \theta)$ and $\underline{\hat{\delta}}=(\hat{\alpha}, \hat{\theta})$. The Newton-Raphson algorithm to maximize the likelihood produces the observed information matrix. Therefore, the variance-covariance matrix is given by

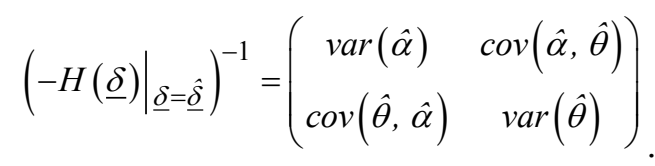

Hence, from the asymptotic normality of MLEs, approximate $100(1-\gamma) \%$ confidence intervals for $\alpha$ and $\theta$ can be constructed as

$$
\hat{\alpha} \pm z_{\gamma / 2} \sqrt{\operatorname{var}(\hat{\alpha})} \text { and } \hat{\theta} \pm z_{\gamma / 2} \sqrt{\operatorname{var}(\hat{\theta})} \text { (14) }
$$

where $z_{\gamma / 2}$ is the upper percentile of standard normal variate. 


\subsection{Computation of MLE}

We have started the iterative procedure by maximizing the log-likelihood function given in equation.(9) directly with an initial guess for $\alpha=0.5$ and $\theta=0.5$, far away from the solution. We have used optim( ) function in R, R Development Core Team (2013) and Rizzo (2008), with option Newton-Raphson method. The iterative process stopped only after 26 iterations. We obtain $\hat{\alpha}=1.838$ and $\hat{\theta}=1.3297$ and the corresponding log-likelihood value is $\ell(\hat{\alpha}, \hat{\theta})=-100.474$. We have plotted the contour plot of $\ell(\alpha, \theta)$ in Figure 3, the $(+)$ indicates the MLE.

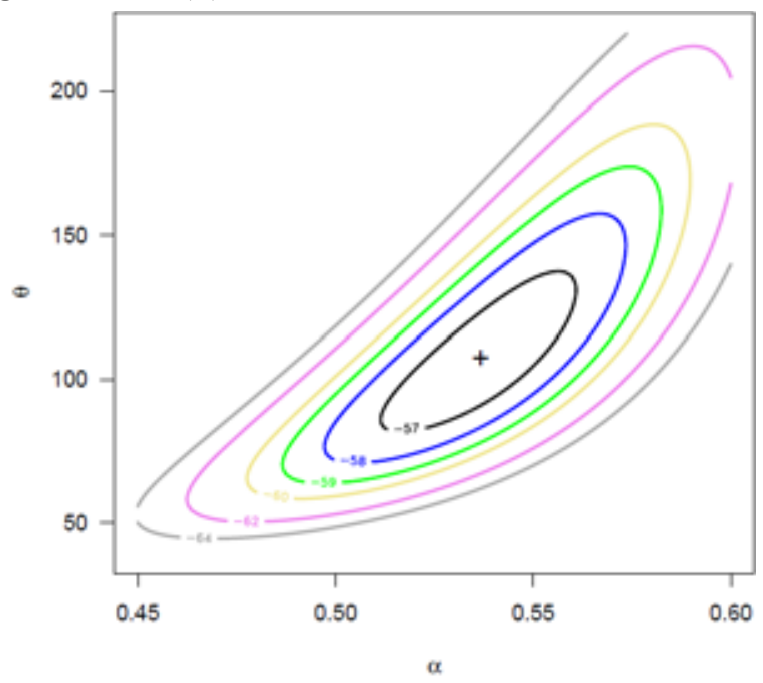

Figure 3 Contour.plot of $\ell(\alpha, \beta)$

The 95\% confidence interval is computed using (13) and (14). The Table 2 shows the ML estimates, standard error(SE) and $95 \%$ Confidence Intervals for the parameters alpha and beta.

Table 2. MLE, standard error and 95\% confidence interval

\begin{tabular}{|c|c|c|c|}
\hline Parameter & MLE & Std. Error & $95 \%$ CI \\
\hline alpha & 1.8381 & 0.27185 & $(1.3053,2.3709)$ \\
\hline Theta & 1.3297 & 0.15382 & $(1.0282,1.6312)$ \\
\hline
\end{tabular}

The Akaike information criterion (AIC) and Bayesian information criterion (BIC) are defined as

$$
\mathrm{AIC}=-2 \ell(\underline{\hat{\delta}})+2 p \text { and } B I C=-2 \ell(\underline{\hat{\delta}})+\mathrm{p} \log (\mathrm{n})
$$

where $\underline{\hat{\delta}}=(\hat{\alpha}, \hat{\theta})$ is the ML estimate of $\underline{\delta}=(\alpha, \beta)$ and $\mathrm{p}$ is the number of parameters estimated in the model. The smaller the value of AIC and BIC, the better the model. The values of the information measures are $\mathrm{AIC}=204.9$ and $\mathrm{BIC}=208.6$, respectively.

\subsection{Model Validation}

To check the validity of the model, we compute the Kolmogorov-Smirnov (KS) distance between the empirical distribution function and the fitted distribution 
function when the parameters are obtained by method of maximum likelihood. The graphical methods Quantile-Quantile (QQ) and Probability-Probability (PP) plots are used for suitability of the model under consideration.

The value of K-S test statistic is 0.0899 and the corresponding $\mathrm{p}$-value is given by 0.8514 . The high $\mathrm{p}$-value clearly indicates that CEP distribution can be used to analyze the given data set, and we have also plotted the empirical distribution function and the fitted distribution function in Figure 4. It is clear that the estimated CEP distribution provides reasonable fit to the given data, Kumar and Ligges (2011).
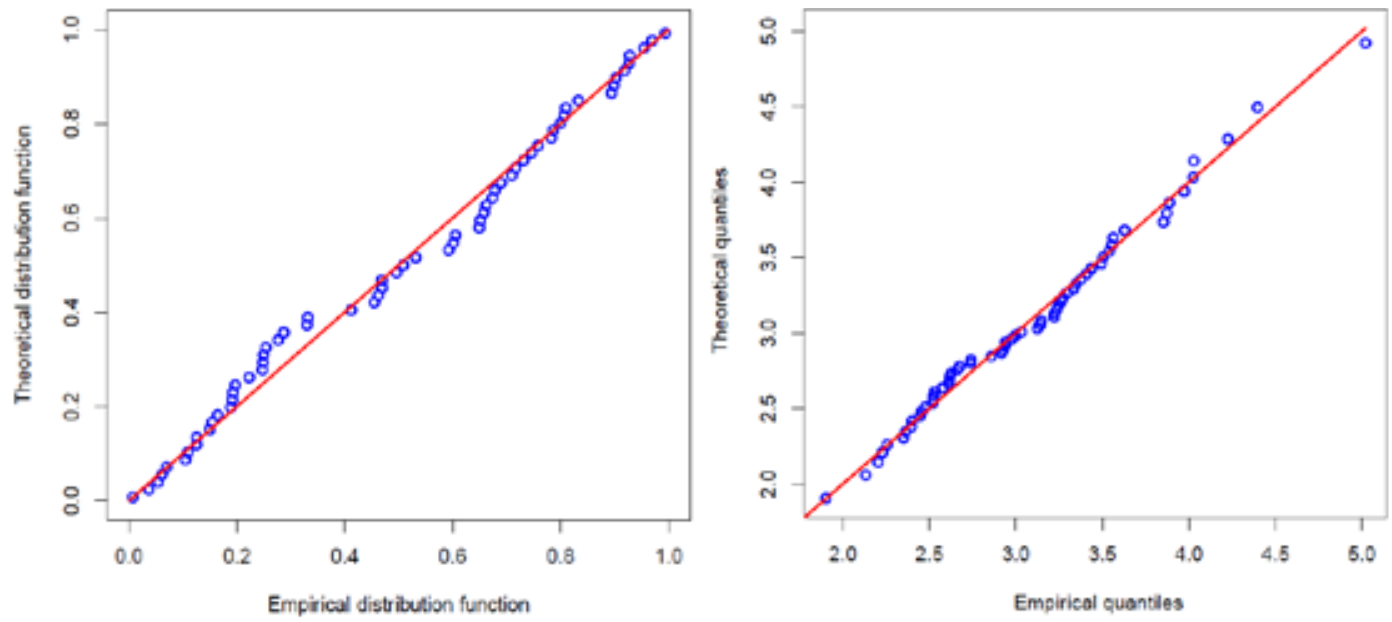

Figure 4 Probability-Probability(PP) plot (top); Quantile-Quantile(QQ) plot (bottom) using MLEs as estimate.

A further support for this finding can be obtained by inspecting the probabilityprobability $(\mathrm{P}-\mathrm{P})$ and quantile-quantile $(\mathrm{Q}-\mathrm{Q})$ plots. The $\mathrm{P}-\mathrm{P}$ plot shows the empirical and theoretical distribution functions. The Q-Q plot shows the estimated versus the observed quantiles. As can be seen from the straight line pattern in Figure 5 the CEP fits the data well.

\section{Bayesian analysis}

Script 5.1 : OpenBUGS script for the Bayesian analysis of CEP distribution Model \{ for $(\mathrm{i}$ in $1: \mathrm{N})\{$

$\mathrm{x}[\mathrm{i}] \sim \mathrm{dCEP} 2$ (alpha, theta) \# CEP distribution reliability $[\mathrm{i}]<-\mathrm{R}(\mathrm{x}[\mathrm{i}], \mathrm{x}[\mathrm{i}]) \quad$ \# to estimate reliability

$\mathrm{f}[\mathrm{i}]<-\operatorname{density}(\mathrm{x}[\mathrm{i}], \mathrm{x}[\mathrm{i}]) \quad$ \# to estimate density

hrf[i] $<-\operatorname{hrf}(x[i], x[i]) \quad$ \# to estimate hazard rate

\# To predict the data set

$\mathrm{ep}[\mathrm{i}]<-(\mathrm{i}-0.5) / \mathrm{N}$

x.new $[\mathrm{i}]<-(1.0 /$ alpha $) * \log (1.0$ - ep[i] $), 1.0 /$ theta $))\}$

\# Prior distributions of the model parameters alpha $\sim \operatorname{dgamma}(0.001,0.001)$ theta $\operatorname{dgamma}(0.001,0.001) \quad\}$

Data 
$\operatorname{list}(\mathrm{N}=63, \mathrm{x}=\mathrm{c}(1.901,2.132,2.203,2.228,2.257,2.350,2.361,2.396,2.397$, $2.445,2.454,2.474,2.518,2.522,2.525,2.532,2.575,2.614,2.616,2.618,2.624$, $2.659,2.675,2.738,2.740,2.856,2.917,2.928,2.937,2.937,2.977,2.996,3.030$, 3.125 , 3.139, 3.145, 3.220, 3.223, 3.235, 3.243, 3.264, 3.272, 3.294, 3.332, 3.346, 3.377, 3.408, 3.435, 3.493, 3.501, 3.537, 3.554, 3.562, 3.628, 3.852, 3.871, 3.886, $3.971,4.024,4.027,4.225,4.395,5.020)$ )

Initial values

list $($ alpha $=0.1$, theta $=10.0) \quad$ \# for Chain 1
$\operatorname{list}($ alpha $=1.0$, theta $=50.0) \quad$ \# for Chain 2

We assume the independent uniform priors for $\alpha \sim G(a, b)$ and gamma prior for $\theta \sim G(c, \mathrm{~d})$ with hyper parameter values $(\mathrm{a}=0.001, \mathrm{~b}=0.001)$ and $(\mathrm{c}=0.001, \mathrm{~d}=0.001)$. We run the model to generate two Markov Chains at the length of 40,000 with different starting points of the parameters. We have chosen initial values $(\alpha=0.1, \theta=10.0)$ for the first chain and $(\alpha=1.0, \theta=50.0)$ for the second chain. The convergence is monitored using trace and ergodic mean plots, we find that the Markov Chain converge together after approximately 2000 observations. Therefore, burnin of 5000 samples is more than enough to erase the effect of starting point(initial values). Finally, samples of size 7000 are formed from the posterior by picking up equally spaced every fifth outcome (to minimize the auto correlation among the generated deviates.), i.e. thin $=5$, starting from 5001.

Therefore, we have the posterior sample $\left(\alpha_{1}^{(j)}, \theta_{1}^{(j)}\right) ; j=1, \ldots, 7000$ from chain 1 and $\left(\alpha_{2}^{(j)}, \theta_{2}^{(j)}\right) ; j=1, \ldots, 7000$ from chain 2 .

The chain 1 is considered for analysis as well as for convergence diagnostics plots. The visual summary is based on posterior sample obtained from chain 1 whereas the numerical summary is presented for both the chains.

\subsubsection{Convergence diagnostics}

Before examining the parameter estimates or performing other inference, it is a good idea to look at plots of the sequential (dependent) realizations of the parameter estimates and plots thereof.

\section{History(Trace) plot}
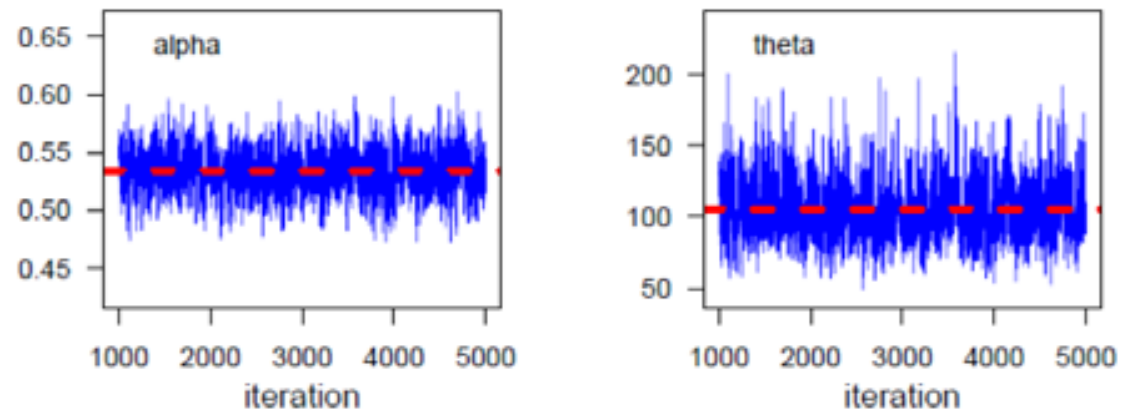

Fig 5.6 Sequential realization of the parameters $\alpha$ and $\theta$. 
We have found that if the Markov chain is not mixing well or is not sampling from the stationary distribution, this is usually apparent in sequential plots of one or more realizations. The sequential plot of parameters is the plot that most often exhibits difficulties in the Markov chain. Figure 5.6 shows the sequential realizations of the parameters of the model. In this case Markov chain seems to be mixing well enough and is likely to be sampling from the stationary distribution.

The plot looks like a horizontal band, with no long upward or downward trends, then we have evidence that the chain has converged.

\section{Running Mean (Ergodic mean) Plot}

Generate a time series(iteration number) plot of the running mean for each parameter in the chain. The running mean is computed as the mean of all sampled values up to and including that at a given iteration. The convergence pattern based on ergodic averages is shown in Figure 5.7 indicating the convergence of the chain.
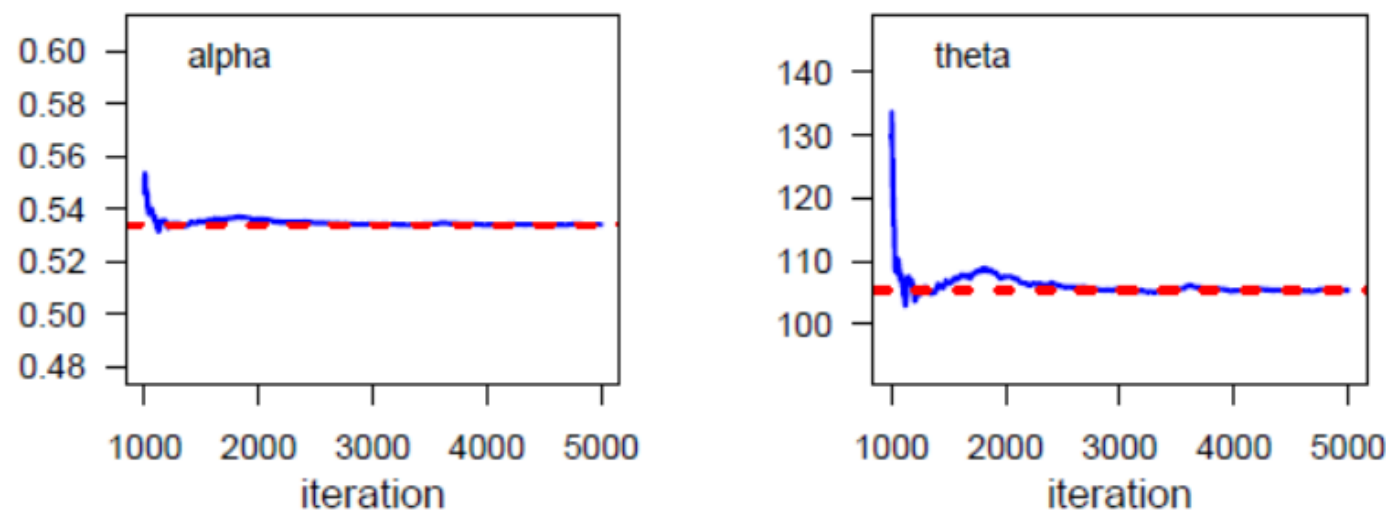

Fig. 5.7 The Ergodic mean plots for alpha and theta.

\section{Brooks-Gelman-Rubin(BGR) diagnostic:}

The Brooks, Gelman and Rubin convergence diagnostic is appropriate for the analysis of two or more parallel chains, each with different starting values which are overdispersed with respect to the target distribution.

OpenBUGS provides the Brooks-Gelman-Rubin statistic for assessing convergence. For a given parameter, this statistic assesses the variability within parallel chains as compared to variability between parallel chains. The model is judged to have convergence if the ratio of between to within variability is close to 1 . The green line represents the between variability, the blue line represents the within variability, and the red line represents the ratio.

Evidence for convergence comes from the red line being close to 1 on the y-axis and from the blue and green lines being stable (horizontal) across the width of the plot. From the Figure 5.8, it is clear that convergence is achieved. Thus, we can obtain the posterior summary statistics. 


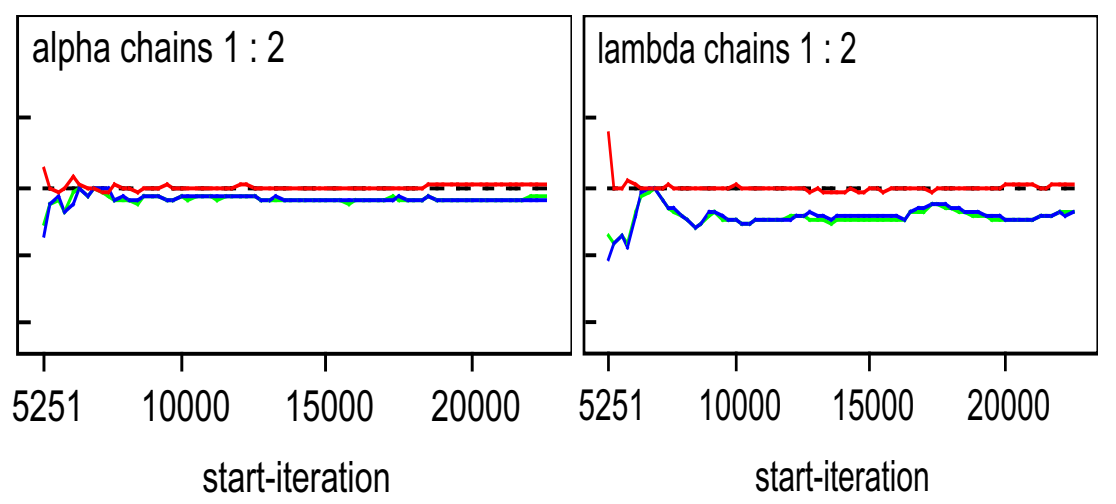

Fig. 5.8 The BGR plots for alpha and theta.

Table 5.2: HPD and Credible intervals

\begin{tabular}{|c|c|c|}
\hline Parameter & Credible Interval & HPD Credible Interval \\
\hline alpha & $(0.4926,0.5745)$ & $(0.4947,0.5758)$ \\
\hline theta & $(68.229,158.605)$ & $(65.99,154.6)$ \\
\hline
\end{tabular}

\subsubsection{Posterior Analysis}

(a) Numerical Summary

The numerical summary is presented for $\left(\alpha_{1}^{(j)}, \theta_{1}^{(j)}\right) ; j=1, \ldots, 7000$ from chain 1 and $\left(\alpha_{2}^{(j)}, \theta_{2}^{(j)}\right) ; j=1, \ldots, 7000$ from chain 2 .

We have considered various quantities of interest and their numerical values based on MCMC sample of posterior characteristics for CEP distribution. The MCMC results of the posterior mean, mode, standard deviation(SD), five point summary statistics (minimum, first quartile, median, third quartile and maximum), 2.5th percentile, 97.5th percentile, skewness, 95\% symmetric and HPD credible intervals of the parameters $\alpha$ and $\theta$ are displayed in Table 5.1 Table 5.1 : Numerical summaries based on MCMC sample of posterior characteristics for CEP2 distribution under gamma priors

\begin{tabular}{|l|c|c|}
\hline \multirow{2}{*}{ Characteristics } & \multicolumn{2}{|c|}{ Chain 1 } \\
\cline { 2 - 3 } Mean & alpha & theta \\
\hline Standard Deviation & 0.53472 & 106.482 \\
\hline Minimum & 0.02092 & 23.318 \\
\hline 2.5th Percentile(P2.5) & 0.44900 & 45.460 \\
\hline First Quartile (Q1) & 0.49260 & 68.229 \\
\hline Median & 0.52090 & 89.908 \\
\hline Third Quartile (Q3) & 0.53500 & 104.200 \\
\hline 97.5th Percentile(P97.5) & 0.54900 & 120.800 \\
\hline Maximum & 0.57450 & 158.605 \\
\hline Mode & 0.60420 & 221.000 \\
\hline Skewness & 0.53400 & 96.964 \\
\hline
\end{tabular}


Highest probability density (HPD): The algorithm described by [Chen and Shao (1999)] is used to compute the HPD intervals under the assumption of unimodal marginal posterior distribution. Table 5.2 shows the HPD and Credible intervals for alpha, and theta parameters.

\section{Visual summary}

(b) Visual summary

The visual graphs include the boxplot, density strip plot, histogram, marginal posterior density estimate and rug plots for the parameters. We have also superimposed the 95\% HPD intervals. These graphs provide almost complete picture of the posterior uncertainty about the parameters. We have used the posterior sample $\left(\alpha_{1}^{(j)}, \theta_{1}^{(j)}\right) ; j=1, \ldots, 7000$ to draw these graphs.

The density strip illustrates a univariate distribution as a shaded rectangular strip, whose darkness at a point is proportional to the probability density, Jackson (2008). Histograms can provide insights on skewness, behaviour in the tails, presence of multi-modal behaviour, and data outliers; histograms can be compared to the fundamental shapes associated with standard analytic distributions.
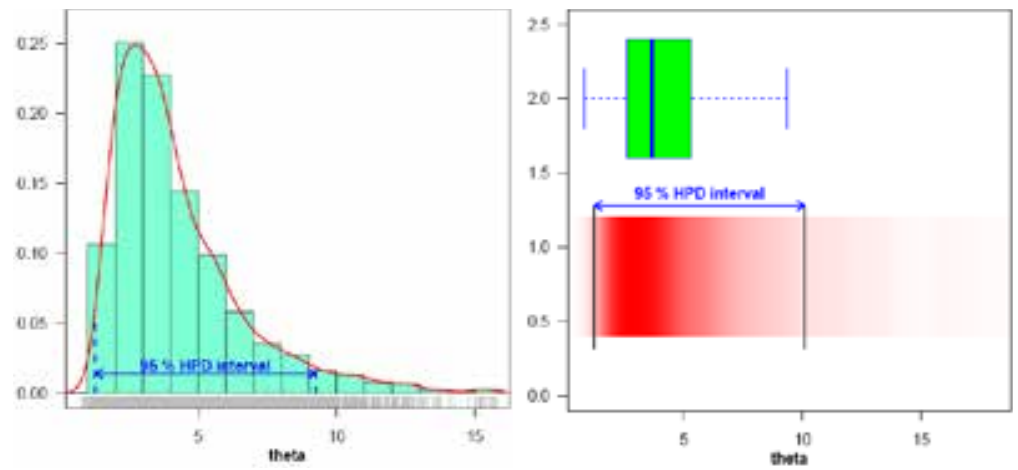

Fig 5.10 Left panel : Histogram, marginal posterior density and 95\% HPD interval ; Right panel : boxplot and density strip of $\theta$, based on posterior sample.
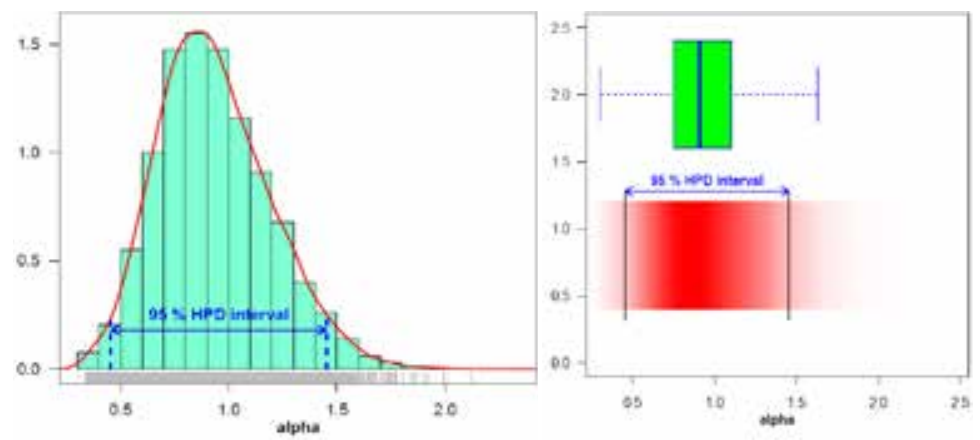

Fig 5.11 Left panel : Histogram, marginal posterior density and 95\% HPD interval; Right panel : boxplot and density strip of $\alpha$, based on posterior sample. 
Figure 5.10 represents the histogram, marginal posterior density and 95\% HPD interval for $\theta$ (left panel) and boxplot and density strip plot (right panel). We have plotted the similar graph for $\alpha$ displayed in Figure 5.11. The kernel density estimates have been drawn using $\mathrm{R}$ with the assumption of Gaussian kernel and properly chosen values of the bandwidths. It can be seen that $\theta$ is symmetric whereas $\alpha$ shows positive skewness.

\subsubsection{Comparison with MLE}

For the comparison with MLE we have plotted three graphs. In Figure 5.13 the density functions $\mathrm{f}(\mathrm{x} ; \hat{\alpha}, \hat{\theta})$ using MLEs and Bayesian estimates, computed via MCMC samples under gamma priors for $\alpha$ and $\theta$, are plotted. It is evident from the Figure, the MLEs and the Bayes estimates are quite close and fit the data very well.

A further support for this finding can be obtained by inspecting the Figure 5.14. In Figure 5.14 we have plotted $2.5^{\text {th }}, 50^{\text {th }}$ and $97.5^{\text {th }}$ quantiles of the estimated density, it can be considered as evaluation of model fit, based on posterior sample,

$$
\left(\alpha_{1}^{(j)}, \theta_{1}^{(j)}\right) ; j=1, \ldots, 7000
$$

We have computed the density function at each observed data point for 7000 posterior samples, using logical function density( ) in OpenBUGS $f\left(x_{i} ; \alpha_{1}^{(j)}, \theta_{1}^{(j)}\right) ; j=1, \ldots, 7000 ; i=1, \ldots, 100$ 产

The density corresponding to MLE has been plotted using the "plug-in" estimates of the parameters. It shows that we have a fairly good model for the given data set.

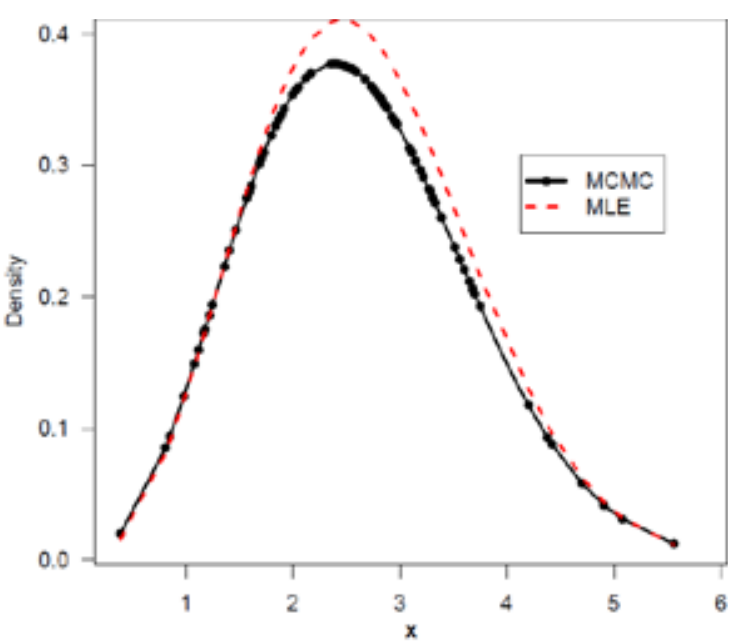

Fig 5.13 The density functions using MLEs and Bayesian estimates, computed via MCMC.

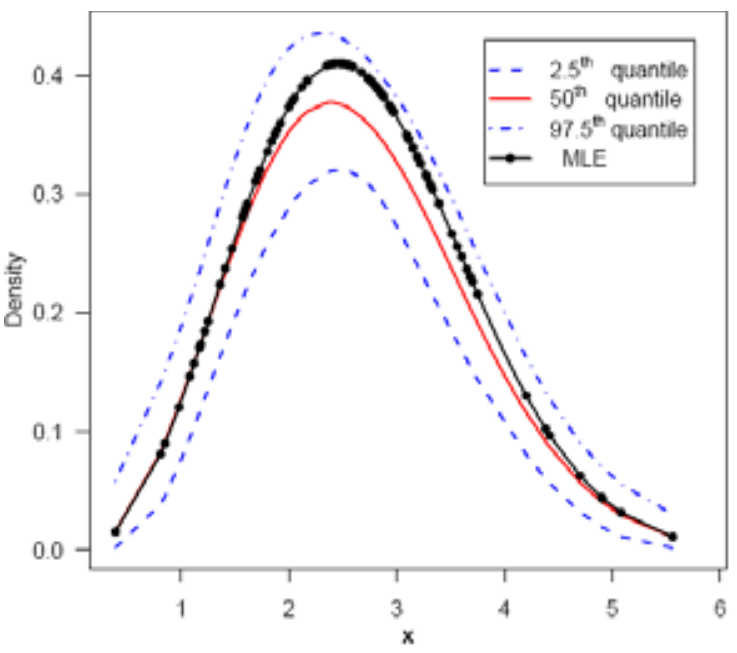

Fig 5.14 Density estimates

\subsubsection{Estimation of reliability function}

In this section, our main aim is to demonstrate the effectiveness of proposed methodology. For this, we have estimated the reliability function using posterior samples. Since we have an effective MCMC technique, we can estimate any function of the parameters. We have used the Kaplan- 
Meier estimate of the reliability function to make the comparison more meaningful. The Figure 5.15, exhibits the estimated reliability function (dashed blue line: $2.5^{\text {th }}$ and $97.5^{\text {th }}$ quantiles; solid red line: $50^{\text {th }}$ quantile) using Bayes estimate based on MCMC output and the empirical reliability function (black solid line). The Figure 5.15 shows that reliability estimate based on MCMC is very close to the empirical reliability estimates.

\subsubsection{Estimation of Hazard and Reliability at $X_{(30)}: \mathbf{t}=\mathbf{2 . 0}$}

The posterior samples may be used

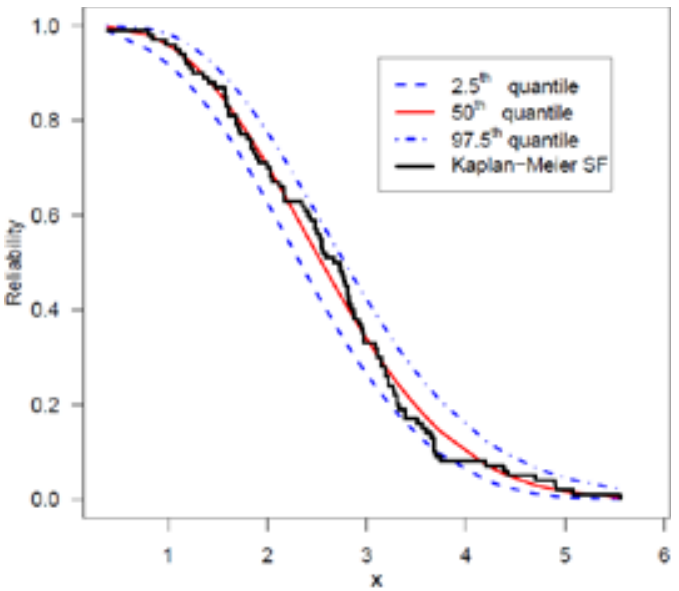

Fig 5.15 Reliability function estimate using MCMC and Kaplan-Meier estimate to completely summarize the posterior uncertainty about the parameters $\alpha$, and $\theta$ through a kernel estimate of the posterior distribution. This is also true of any function of the parameters e.g. reliability and hazard functions. Suppose we wish to give point and interval estimates for reliability and hazard functions at the mission time $t=2.0$ (at the 30th observed data point).

We have computed the hazard and reliability functions at mission time $\mathrm{t}=2.0$ (at the 30th observed data point) for 7000 posterior samples, using logical function hrf ( ) and reliability( ), [Kumar et al. (2010)] in OpenBUGS. It can be computed directly using hazard and reliability functions given in (5.2.4) and (5.2.3) respectively

$$
\begin{aligned}
& h\left(x=2.0 ; \alpha_{1}^{(j)}, \theta_{1}^{(j)}\right) ; j=1, \ldots, 7000 \text { and } \\
& R\left(x=2.0 ; \alpha_{1}^{(j)}, \theta_{1}^{(j)}\right) ; j=1, \ldots, 7000
\end{aligned}
$$

Alternatively, we can use $\mathrm{R}$ functions hcom.exp.power() and scom.exp.power() given in Appendix-A as A.5.2.
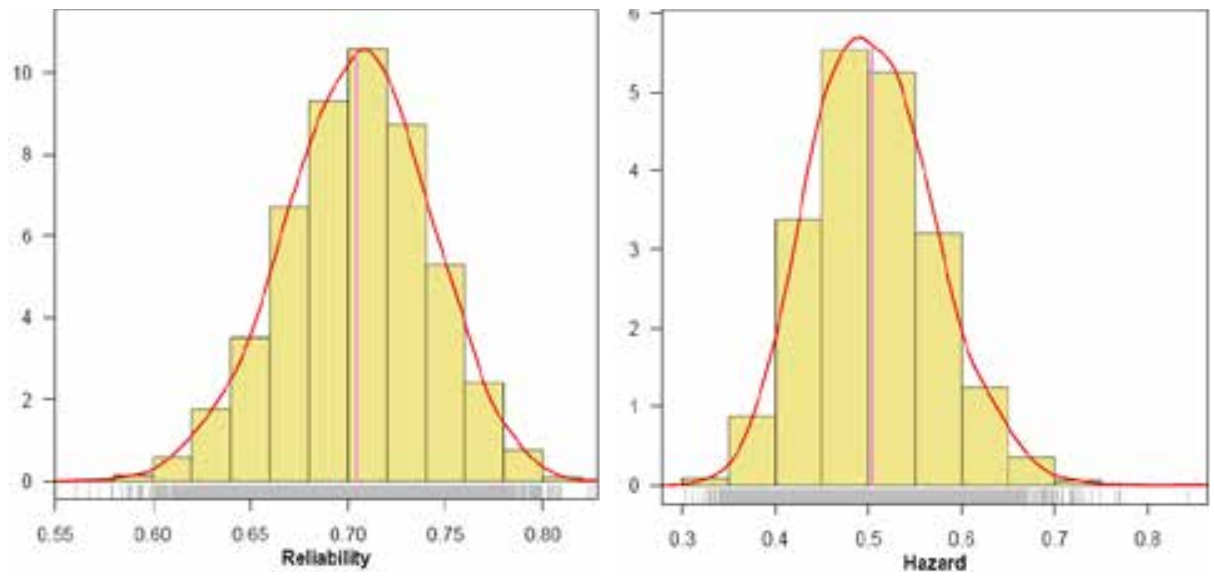

Fig 5.15 Visual summary of reliability(left panel) and hazard(right panel) at $t=2.0$ 
The marginal posterior density estimates of the reliability (left panel) and hazard functions(right panel) and their histograms based on samples of size 7000 are shown in Figure. 5.15 using the Gaussian kernel.

Table 5.3: Posterior summary for Reliability and Hazard functions at $t=2.0$

\begin{tabular}{|l|c|c|}
\hline \multicolumn{1}{|c|}{ Characteristics } & Reliability & Hazard \\
\hline Mean & 0.7043 & 0.5047 \\
\hline Standard Deviation & 0.0376 & 0.0669 \\
\hline Minimum & 0.5607 & 0.3041 \\
\hline 2.5th Percentile(P2.5) & 0.6272 & 0.3846 \\
\hline First Quartile (Q1) & 0.6791 & 0.4567 \\
\hline Median & 0.7054 & 0.5013 \\
\hline Third Quartile (Q3) & 0.7299 & 0.5484 \\
\hline 97.5th Percentile(P97.5) & 0.7755 & 0.6451 \\
\hline Maximum & 0.8259 & 0.8423 \\
\hline Mode & 0.7083 & 0.4893 \\
\hline Skewness & -0.1501 & 0.2992 \\
\hline 95\% Credible Interval & $(0.6272,0.7755)$ & $(0.3846,0.6451)$ \\
\hline 95\% HPD Credible Interval & $(0.6313,0.7788)$ & $(0.3770,0.6356)$ \\
\hline
\end{tabular}

It is evident from Figure 5.15 the estimates that the marginal distribution of reliability is negatively skewed whereas hazard is positively skewed.

The MCMC results of the posterior mean, mode, standard deviation(SD), five point summary statistics (minimum, first quartile, median, third quartile and maximum), 2.5th percentile, 97.5th percentile, skewness, 95\% symmetric and HPD credible intervals of reliability and hazard functions are displayed in Table 4.5. The ML estimates of reliability and hazard function at $t=2.0$ are computed using invariance property of the MLE. ML estimates $\hat{\mathrm{h}}(\mathrm{t}=2.0)=0.5264$ and $\hat{\mathrm{R}}(\mathrm{t}=2.0)=0.7097$.

A trace plot is a plot of the iteration number against the value of the draw of the parameter at each iteration. Figure 5.16 display 7000 chain values for the hazard $\mathrm{h}(\mathrm{t}=2.0)$ and reliability $\mathrm{R}(\mathrm{t}=2.0)$ functions, with their sample median and $90 \%$ credible intervals.
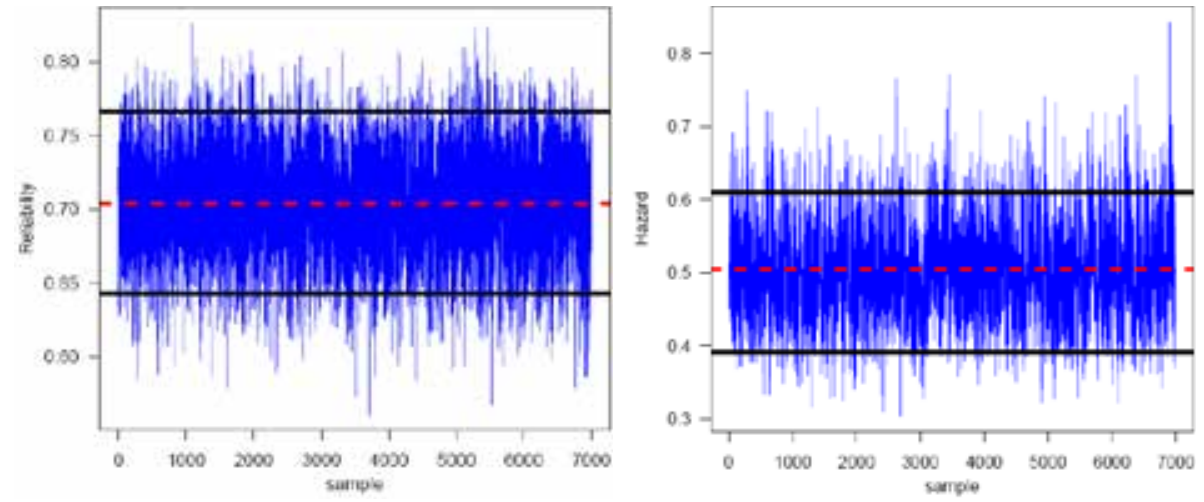

Fig 5.16 MCMC output of $R(t=2.0)$ and $h(t=2.0)$. Dashed line $(. .$.$) represents the posterior median$ and solid lines(-) represent lower and upper bounds of $90 \%$ probability intervals(HPD) 


\subsubsection{Model compatibility}

\section{Posterior Predictive Checks:}

Once we have determined that the algorithm converged to the appropriate distribution and mixed well so that we have an adequate sample from the posterior distribution, our next step should be to determine whether the model fit the data well enough to justify drawing inference about the parameters. At this point, if we have estimated several different models, we can also begin to decide which is the best model.

One of the best and most flexible approaches to examining model fit is the use of posterior predictive distributions, [Gelman (2003)] and [Gelman et al. (2004)]. The posterior predictive distribution for a model is the distribution of future observations that could arise from the model under consideration. The posterior predictive distribution takes into account both (1) parametric uncertainty and (2) sampling uncertainty from the original model. Parametric uncertainty is captured via the posterior distribution for the parameters, a sample of which is the result of simulation using MCMC methods. Sampling uncertainty is captured via the specification of the sampling density for the data. Thus, we can simulate data from the posterior predictive distribution, compare it with the observed data, and, if the simulated data are similar to the observed data, we may conclude the model fits well.

Implementation of posterior predictive simulation is relatively simple, given an MCMC-generated sample of size 2000 from the posterior distribution for the parameters in a model $\underline{\delta}=(\alpha, \theta)$, and can often be incorporated as part of the MCMC algorithm itself. For each value of $\underline{\delta}$ simulated from the posterior, we generate a new observation from the sampling distribution for the data, using that parameter value, for every original observation in the sample. Thus, we have

$$
x_{i}^{r e p} ; i=1, \ldots, 100 \text { for each }\left(\alpha_{1}^{(j)}, \theta_{1}^{(j)}\right) ; j=1, \ldots, 1000
$$

In fact, we have predicated the entire data set and we have 1000 replications of each $x_{i} ; i=1, \ldots, 100$. We view the model-checking as a comparison of the data with the replicated data given by the model, which includes exploratory graphics. In fact statistical graphics provides implicit or explicit model checks. Figure 5.17 represents the Q-Q plot of predicted quantiles vs. observed quantiles. We, therefore, conclude that the Gompertz extension model is compatible with the given data set.

To obtain further clarity on our conclusion for the study of model compatibility, we have considered

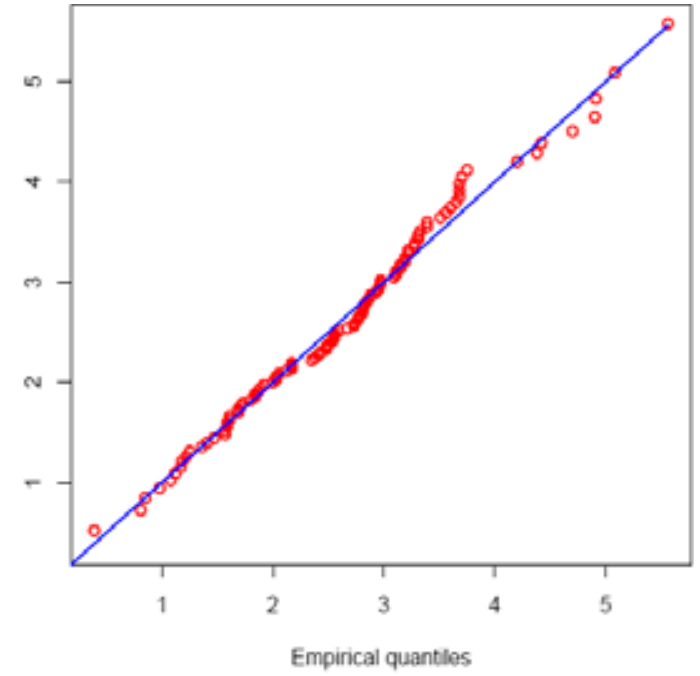

Fig. 5.17 Q-Q plot of predictive quantiles versus empirical quantiles 
plotting of density estimates of smallest, largest and 30th smallest i.e. $\left(\mathrm{X}_{(1)}, \mathrm{X}_{(100)}\right.$ and $\mathrm{X}_{(30)}$ replicated future observations from the model with superimposed corresponding observed data. For this purpose, 2000 samples have been drawn from the posterior using MCMC procedure and then obtained predictive samples from the model under consideration using each simulated posterior sample. The size of predictive samples is same as that of observed data.

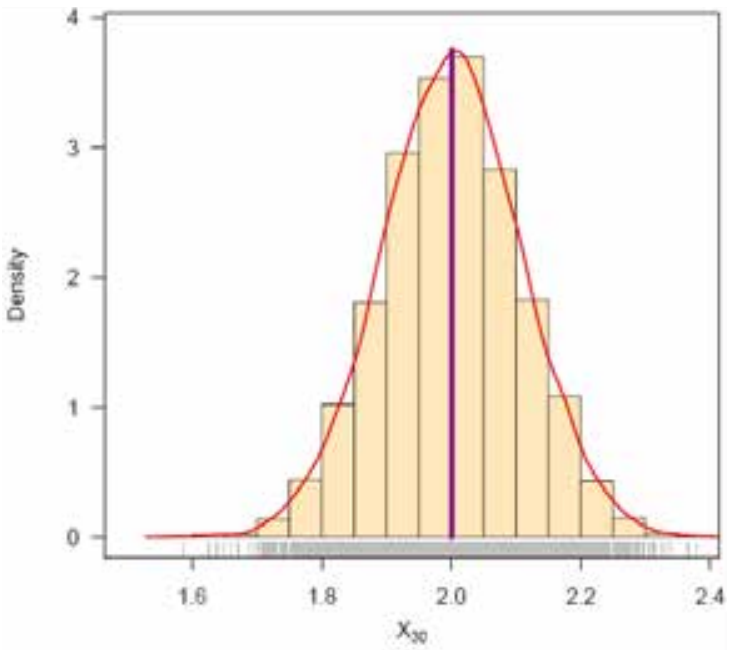

Fig 5.18 Density estimates of the $X_{(30)}$, vertical lines represent corresponding observed values
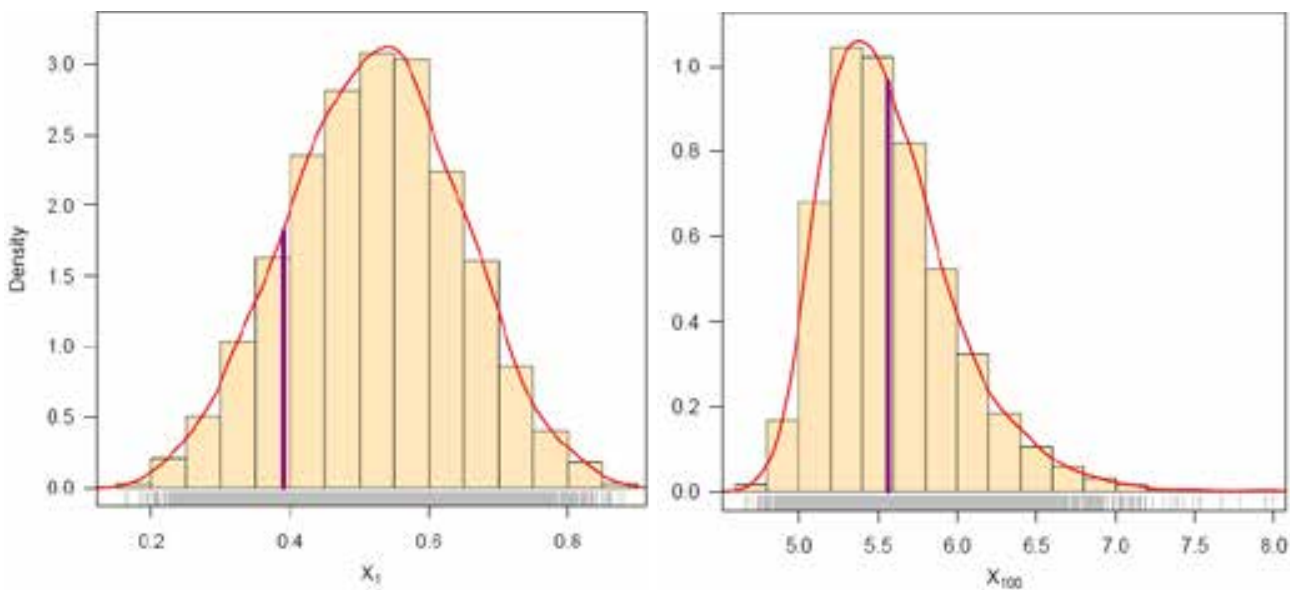

Fig 5.19 Density estimates of the smallest $\left(X_{(1)}\right)$ and largest $\left(X_{(100)}\right)$ order future observations, vertical lines represent corresponding observed values

Density estimates based on replicated future data sets are shown in Figures 5.18 and 5.19. Figure 5.19 represents the estimates corresponding to smallest and largest predictive observations, whereas the same for 30th smallest observations is shown in Figure 5.18. The corresponding observed values are also shown by means of vertical lines.

As the Figures 5.18 and 5.19 show, the posterior predictive distributions are centered over the observed values, which indicates good fit. In general, the distribution of replicated data appears to match that of the observed data fairly well.

The MCMC results of the posterior mean, median, mode of smallest and largest $\left(\mathrm{X}_{(1)}\right.$ and $\left.\mathrm{X}_{(100)}\right)$ and $\mathrm{X}_{(30)}$ are displayed in Table 5.4. 
Table 5.4: Posterior characteristics

\begin{tabular}{|c|c|c|c|c|}
\cline { 2 - 5 } \multicolumn{1}{c|}{} & Observed & Mode & Mean & Median \\
\hline $\mathrm{X}_{(1)}$ & 0.39 & 0.545 & 0.521 & 0.606 \\
\hline $\mathrm{X}_{(30)}$ & 2.00 & 2.007 & 2.001 & 2.072 \\
\hline $\mathrm{X}_{(100)}$ & 5.56 & 5.378 & 5.580 & 5.803 \\
\hline
\end{tabular}

Figure 5.20 exhibits graphical posterior predictive check of the model adequacy, solid line( ) represents the posterior median and dashed lines (...) represent lower and upper bounds of $95 \%$ probability intervals, observed data is superimposed. The predictive data reflect the expected observations after replicating the experiment in future, having already observed $\mathrm{x}$ and assuming that the adopted model is true.

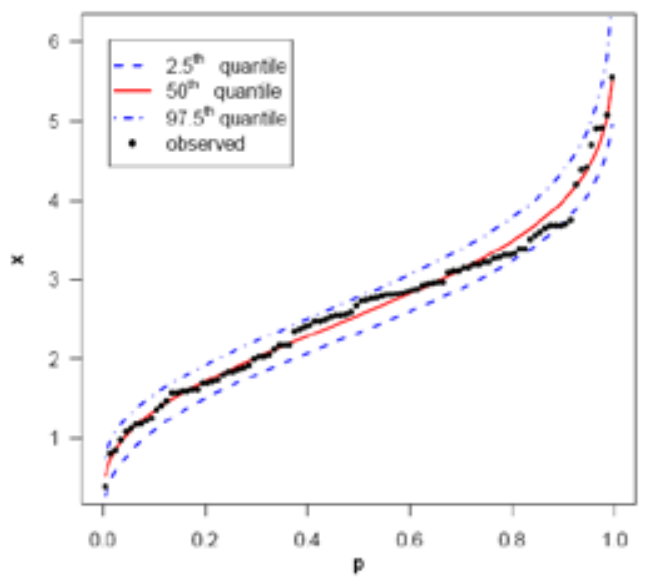

Fig. 5.20 Graphical posterior predictive check of the model adequacy.

Overall, the results of the posterior predictive simulation indicate that model fits these data particularly well. Model fit assessments based on posterior predictive checks are somewhat too liberal, and posterior predictive checks should not be used for model selection [Ntzoufras (2009)].

\section{Conclusions}

We have discussed the Markov chain Monte Carlo (MCMC) method to compute the Bayesian estimates the parameters and reliability functions of exponentiated loglogistic distribution based on a complete sample. We have obtained the probability intervals for parameters. The MCMC method provides an alternative method for parameter estimation of the exponentiated log-logistic distribution. It is more flexible when compared with the traditional methods such as MLE method. Moreover, 'exact' probability intervals are available rather than relying on estimates of the asymptotic variances. Indeed, the MCMC sample may be used to completely summarize posterior distribution about the parameters, through kernel estimation. This is also true for any function of the parameters such as reliability and hazard functions. We have applied the developed techniques on a real data set. The paper successfully describes the scope 
of Markov chain Monte Carlo (MCMC) technique in the exponentiated log-logistic distribution. Thus, the tools developed can be applied for full Bayesian analysis of complementary exponential power distribution.

\section{References}

Albert, J. (2009). Bayesian Computation with R. 2nd edition. Springer, New York.

Balakrishnan, N. \& Malik, H.J. (1987). Moments of order statistics from truncated log-logistic distribution. Journal of Statistical Planning and Inference. 17: 251-267.

Bennette, S. (1983). Log-logistic regression models for survival data. Applied Statistics. 32: 165 171.

Chen, M. H. \& Shao, Q. M. (1999). Monte Carlo estimation of Bayesian credible intervals and HPD intervals. Journal of Computational and Graphical Statistics. 8(1): 69-92.

Chhikara, R.S. and Folks, J.L. (1977). The inverse Gaussian distribution as a lifetime model. Technometrics. 19: 461-468.

Gelfand, A.E. \& Smith, A.F.M. (1990). Sampling based approach to calculating marginal densities. Journal of the American Statistical Association. 85: 398-409.

Gelman, A.; Carlin, J.; Stern, H., and Rubin, D. (2004). Bayesian Data Analysis. Second Edition, Chapman \& Hall, London.

Geman, S. \& Geman, D. (1984). Stochastic Relaxation, Gibbs Distributions, and the Bayesian Restoration of Images. IEEE Transactions of Pattern Analysis and Machine Intelligence. 6: 721741.

Gupta, R. D. \& Kundu, D. (1999). Generalized exponential distributions. Australian and New Zealand Journal of Statistics. 41(2): 173 - 188.

Hamada, M.S.; Wilson, A.G.; Reese, C.S. and Martz, H.F.(2008). Bayesian Reliability. Springer, New York.

Hoff, P.D. (2009). A First Course in Bayesian Statistical Methods. Springer, New York.

Howlader, H.A. \& Weiss, G.(1992). Log-logistic survival estimation based on failure-censored data. Journal of Applied Statistics. 19, No. 2 : 231-240.

Klugman, S.; Panjer, H. \& Willmot, G. (2012) Loss Models: From Data to Decisions. (4th edition). John Wiley \& Sons, New York.

Kumar, V. (2010). Bayesian analysis of exponential extension model. J. Nat. Acad. Math. 24 :109128.

Kumar, V. \& Ligges, U. (2011). reliaR : A package for some probability distributions. http://cran.rproject.org/web/packages/reliaR/index.html.

Kumar, V.; Ligges, U. \& Thomas, A. (2010). ReliaBUGS User Manual : A subsystem in OpenBUGS for some statistical models. version 1.0, OpenBUGS 3.2.1, http://openbugs.info/w/Downloads/

Lawless, J. F., (2003). Statistical Models and Methods for Lifetime Data. 2nd ed., John Wiley and Sons, New York.

Lee, E.T. \& Wang, T.W.(2003). Statistical Methods for Survival Data Analysis. 3rd ed., John Wiley \& Sons.

Lunn, D.(2010). Recent Developments in the BUGS software. ISBA Bulletin. 17(3):16-17.

Lunn, D.J., Andrew, A., Best, N. \& Spiegelhalter, D. (2000). WinBUGS - A Bayesian modeling framework: Concepts, structure, and extensibility. Statistics and Computing. 10: 325-337

Lunn, D.J.; Jackson, C.; Best, N.; Andrew, A., \& Spiegelhalter, D. (2013). The BUGS Book : A Practical 
Introduction to Bayesian Analysis. Chapman \& Hall/CRC, London.

Marshall, A. W. \& Olkin, I. (1997). A new method for adding a parameter to a family of distributions with application to the exponential and Weibull families. Biometrika. 84(3) : 641-652.

Marshall, A. W. \& Olkin, I. (2007). Life Distributions: Structure of Nonparametric, Semiparametric and Parametric Families. Springer, New York.

Mudholkar, G.S. \& Srivastava, D.K. (1993). Exponentiated Weibull family for analyzing bathtub failure-rate data. IEEE Transactions on Reliability. 42(2): 299-302.

Murthy, D.N.P., Xie, M. \& Jiang, R. (2004). Weibull Models. Wiley, New York.

Nadarajah, S. \& Kotz, S. (2006). The exponentiated type distributions. Acta Applicandae Mathematicae. 92: 97-111.

Ntzoufras, I. (2009). Bayesian Modeling using WinBUGS. John Wiley \& Sons, New York.

R Development Core Team (2013). R: A language and environment for statistical computing. R Foundation for Statistical Computing. Vienna, Austria. ISBN 3-900051-07-0, URL http:// www.R-project.org.

Rizzo, M. L.(2008). Statistical computing with R. Chapman \& Hall/CRC.

Rosaiah, K.; Kantam, R.R.L. \& Kumar, Ch. S. (2006). Reliability test plans for exponentiated loglogistic distribution. Economic Quality Control. 21(2) : 165-175.

Rosaiah, K.; Kantam, R.R.L. \& Santosh Kumar, Ch. (2007). Exponentiated log-logistic distribution an economic reliability test plan. Pakistan Journal of Statistics. 23 (2) : $147-156$.

Santana, T.V.F.; Ortega, E.M.M.; Cordeiro, G.M. \& Silva, G.O. (2012). The Kumaraswamy-Log-Logistic Distribution. Journal of Statistical Theory and Applications. 11(3) : 265-291.

Srivastava, P.W. \& Shukla, R. (2008). A Log-Logistic Step-Stress Model. IEEE Transactions on Reliability. 57(3) : 431-434.

Tadikamalla, P. R. and Johnson, N.L. (1982). Systems of frequency curves generated by the transformation of logistic variables. Biometrika. 69: 461-465.

Thomas, A.; O'Hara, B., Ligges, U. and Sturtz, S. (2006). Making BUGS Open. R News. 6: 12-17.

Thomas, A.(2010). OpenBUGS Developer Manual. ver.3.1.2, http://www.openbugs.info/

Tukey, J.W. (1977). Exploratory Data Analysis. Reading, Mess. Addison-Wesley. 